\title{
EFFECT OF GONADOTROPINS ON OVULATION AND OVARIAN HISTOLOGY IN THE
}

IMMATURE MONGOLIAN GERBIL (1)

THEODORE V. FISCHER AND DON LOWELL FISHER

Department of Anatomy, The University of

Michigan, Ann Arbor, Michigan 48104

\begin{abstract}
The present study is an analysis of the effects of superovulatory doses of gonadotropins on the rate and time of ovulation and ovarian histology in immature gerbils. Groups were treated with various combinations of pregnant mare serum gonadotropin (PMSG) and human chorionic gonadotropin (HCG). The maximum superovulatory response followed treatment with 10 IU PMSG and 20 IU HCG. High dosages of PMSG inhibited superovulation, as did some combinations involving the highest dose of HCG. Entrapment of ova within corpora lutea was common in groups receiving high doses of either gonadotropin. Luteal regression, appearing by day 3 , occurred often in groups receiving high doses of HCG. A dose of 10 IU PMSG and 5 IU HCG resulted in both a near maximal superovulatory response and the least abnormal ovarian alteration.
\end{abstract}

There is a wealth of literature detailing the action of gonadotropins on ovulation itself, but 1ittle attention has been given to their ovarian effects. Wilson and Zarrow ('62) report that in the rat high doses of PMSG inhibit ovulation due to the formation of cystic follicles, and that in the mouse rapid follicular luteinization may entrap many ova. In the rat, cole ('36) demonstrated that high PMSG doses induce a similar early luteinization with entrapment of ova. In the calf, Larsen et al. ('71) reported normal luteal formation and regression following LH treatment.

Reproductive studies in the Mongolian gerbil have included investigations of the estrous cycle (Barfield and Beeman, '68), ovulation, fertilization and cleavage stages (Marston and Chang, '66). Superovulation was also briefly mentioned as part of that experimental work and in an introductory study of our own 
(Fischer and Fisher, '73). There is no published report on the effects of gonadotropins on the histology of the gerbil ovary.

This study was undertaken to establish the necessary dosages of PMSG and HCG for maximum superovulation in the immature gerbil, and to study the ovarian histology of the treated animals.

MATERIALS AND METHODS Four- to six-week old prepuberal Mongolian gerbils were placed in eighteen groups of four each (table 1) and were given intraperitoneal injections of from 0-30 IU PMSG in $1 / 2 \mathrm{ml}$ of $0.9 \%$ saline followed 50 hours later by a similar injection of 0-30 IU HCG. Ovulation was ascertained by tearing apart and/or flushing the oviduct. Mean ovulation rates of each group were compared by the Student-Neuman-Keuls test (Snedecor and Cochran, '67) to that of the group having the highest rate.

Ovaries were fixed in formalin-alcohol-acetic acid, sectioned and stained with hematoxylin and eosin.

RESULTS The effects on ovulation of two independent variables, PMSG and HCG, were tested at various dosage levels (table 1). Neither hormone alone was sufficient to stimulate ovulation. High doses (30 IU) of PMSG significantly inhibited superovulation, although many ova were released in all other treatment groups. The best response was obtained in the group receiving 10 IU PMSG and 20 IU HCG. Significantly lower than this group $(p<0.05)$ were the ovulation rates in groups receiving the highest HCG dose ( 30 IU) and 5 and 30 IU of PMSG.

Sections of ovaries from all treatment groups were compared with ovaries of non-treated immature gerbils, mature pregnant and non-pregnant gerbils, and gonadotropin-treated immature mice.

Some oocytes were trapped within corpora lutea in each group. However, an uncommonly large number of corpora lutea contained oocytes in groups receiving the higher dosages (20 and $30 \mathrm{IU}$ ) of both PMSG and HCG. Such entrapment was never 
TABLE ]

Average number of ova shed in response to PMSG and HCG injections in the Mongolian gerbila

\begin{tabular}{|c|c|c|c|c|c|}
\hline \multirow{2}{*}{$\begin{array}{c}\text { PMSG Dosage } \\
\quad \text { (IU) }\end{array}$} & \multicolumn{5}{|c|}{ HCG Dosage (IU) } \\
\hline & 0 & 5 & 10 & 20 & 30 \\
\hline 0 & & & $0.0 *$ & & \\
\hline 5 & & $18.5 \pm 7.8^{b}$ & $26.3 \pm 4.1$ & $25.0 \pm 3.6$ & $9.0 \pm 6.8 *$ \\
\hline 10 & & $29.3 \pm 3.3$ & $28.3 \pm 4.8$ & $33.3 \pm 3.9$ & $18.8 \pm 3.9$ \\
\hline 20 & $0.0 *$ & $13.8 \pm 6.4^{*}$ & $25.0 \pm 8.4$ & $19.3 \pm 71.9$ & $21.0 \pm 5.8$ \\
\hline 30 & & $0.3 \pm 0.2^{\star}$ & $4.8 \pm 4.7 *$ & $0.5 \pm 0.4^{*}$ & $0.0 *$ \\
\hline
\end{tabular}

\footnotetext{
Means of each group were compared with that of the group having the highest ovulation rate (10 IU PMSG, 20 IU HCG).

${ }^{b}$ Each value represents the mean of four observations \pm SEM

* $p<0.05$
}

observed in corpora lutea of mature, non-treated gerbils, but occasionally occurred in the immature treated mice. In some cases, up to $90 \%$ of the corpora lutea in an ovary contained an entrapped egg. In such ovaries, several entrapped ova may be seen in a single section (fig. 1).

Early luteal involution occurred in several groups. As early as day 3 all corpora lutea had regressed in some animals (fig. 2). Many luteal cells showed typical degenerative changes as macrophages and fibroblasts invaded the corpus luteum, but other intact luteal cells retained their normal vesicular nuclei and tended to elongate. The cellular outlines became obscured and the cells assumed an irregularly branched or spindle-shaped appearance. The combination of cellular infiltration and clusters of altered luteal cells gave the corpus luteum a distinctive "swirled" appearance (fig. 3). Entrapped ova were noted in these as well as in normal corpora lutea (figs. 4, 5).

DISCUSSION Treatment with a broad dosage range of both PMSG and HCG in many combinations led to adequate but variable superovulation in the immature 
gerbi1. The greatest range ( 0 to 59 ova) occurred in the group receiving 20 IU of both hormones. Even though several treatment groups showed significantly lower rates than the optimal (10 IU PMSG, 20 IU HCG), they still fell near or within the arbitrary level considered an adequate superovulatory response (20 ova). However, 30 IU PMSG is inhibitory since all groups receiving this level showed a severe depression of the ovulation rate. This finding is in contrast to an earlier study in the mature gerbil, in which even higher PMSG dosages resulted in superovulation (Marston and Chang, '66).

When selecting the best hormonal regimen from a series of combinations which all seem adequate, other factors ought to be considered. Moderate or high doses of HCG resulted in early regression of corpora lutea in at least $50 \%$ of the ovaries so treated. Only with the lowest HCG level was this abnormality kept to a minimum. In addition, in groups receiving the two highest levels of both hormones, large numbers of ova were entrapped. The least number of included ova occurred in groups receiving 5 IU HCG and 5 or 10 IU PMSG. It is important to choose a hormonal dosage which both reduces these two effects and allows adequate superovulation. Of the possibilities, the hormonal regimen of choice would be 10 IU PMSG and 5 IU HCG.

\section{LITERATURE CITED}

Barfield, M. A., and E. A. Beeman 1968 The oestrus cycle in the Mongolian

\section{FIGURE LEGENDS}

1 Section of an ovary 3 days after HCG administration, showing normal corpora lutea. Three entrapped ova are present (arrows). X 56.

2 Section of an ovary 3 days after HCG administration, showing luteal regression. $\times 56$.

3 Higher magnification of a regressing corpus luteum. Pyknotic nuclear fragments and spindle-shaped luteal cells with irregular or ovoid nuclei predominate. $\times 404$.

4 Entrapped oocyte in a regressing corpus 1uteum. X 404.

5 Entrapped oocyte in a normal corpus luteum. $\times 404$. 

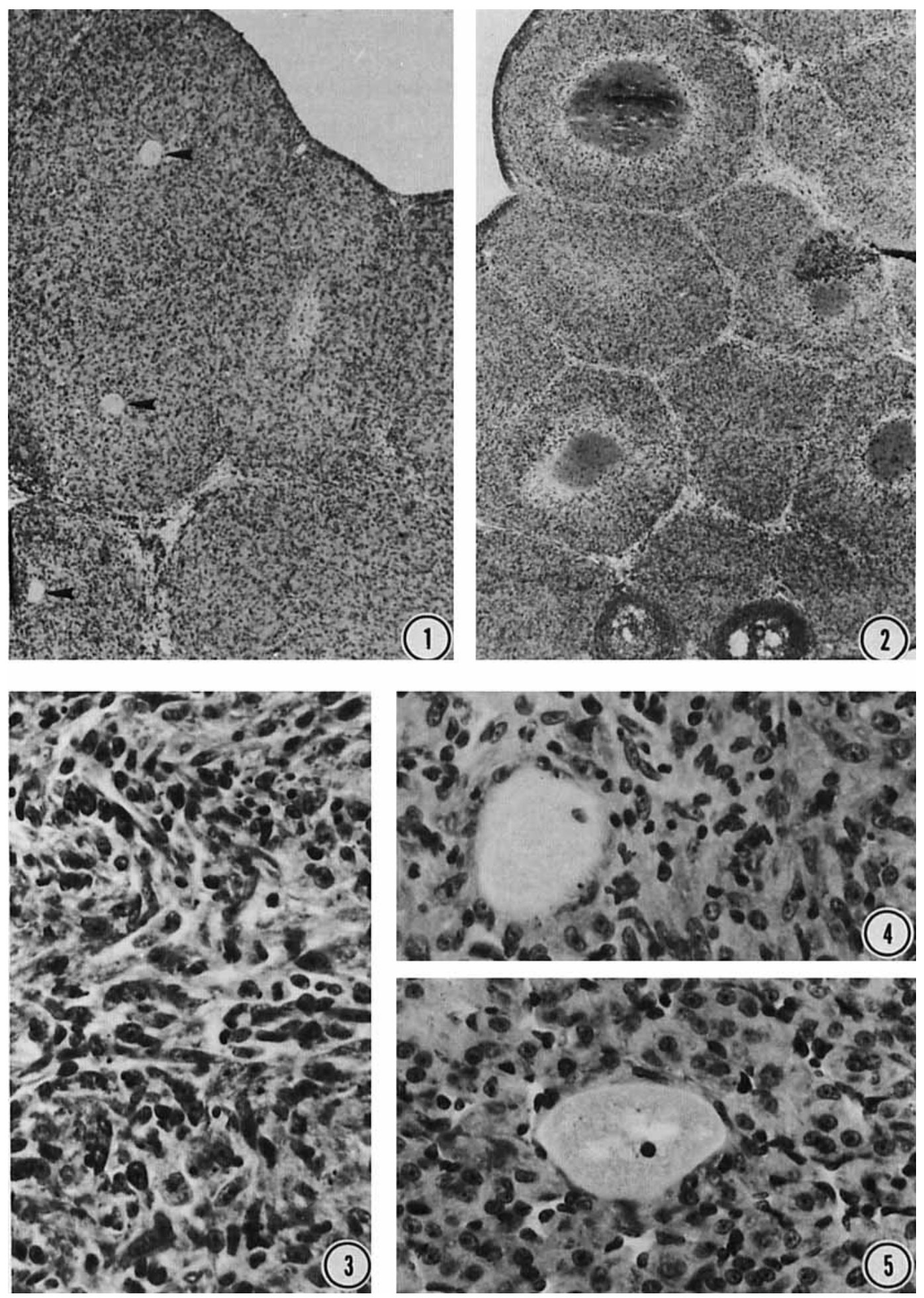
gerbil, Meriones unguiculatus. J. Reprod. Fert., 17: 247-251.

Cole, H. H. 1936 on the biological properties of mare gonadotrophic hormone. Am. J. Anat., 59: 299-331.

Fischer, T. V., and D. L. Fisher 1973 Superovulation in the Mongolian gerbil. Anat. Rec., 175: 319.

Larsen, L. L., G. E. Seidel and R. H. Foote 1971 Anatomical and histological observations on the reproductive organs in superovulated calves. J. Anim. Sci., 33: 799-803.

Marston, J. H., and M. C. Chang 1966 The morphology and timing of fertilization and early cleavage in the Mongolian gerbil and deer mouse. J. Embryo1. Exp. Morph., 15: 169-191.

Snedecor, G. W., and W. G. Cochran 1967 Statistical Methods. Sixth edition, The Iowa State University Press, Ames, Iowa.

Wilson, E. D., and M. X. Zarrow 1962 Comparison of superovulation in the immature mouse and rat. J. Reprod. Fertil., 3: 148-158.

\section{REFERENCE}

1 This study was supported in part by Institutional Research Grant IN-40N to The University of Michigan from the American Cancer Society. 